\title{
Association between Sleep Deprivation and Metformin Treatment on Pancreatic and Liver Function in Mice
}

Srinivasan Periasamy ${ }^{1}$, Se-Ping Chien ${ }^{2}$, Chen Hsu ${ }^{1}$, Dur-Zong Hsu ${ }^{1,}{ }^{*}$, Ming-Yie Liu ${ }^{1,}{ }^{*}$

1. Department of Environmental and Occupational Health, College of Medicine, National Cheng Kung University, 138 Sheng-Li Rd., Tainan, Taiwan; E-Mails: srini.ap@gmail.com; vmtp8271@hotmail.com; duroznghsu@gmail.com; myliu@mail.ncku.edu.tw

2. Department of Food and Beverage Services, Tainan University of Technology, 529 JhongJheng Rd., Yong Kang, Tainan, Taiwan; E-Mail: t10062@mail.tut.edu.tw

* Correspondence: Dur-Zong Hsu, Ming-Yie Liu; E-Mails: durzonghsu@gmail.com; myliu@mail.ncku.edu.tw

Academic Editor: Tatsuo Kanda

OBM Hepatology and Gastroenterology

2020, volume 4, issue 2

doi:10.21926/obm.hg.2002047
Received: March 10, 2020

Accepted: May 14, 2020

Published: May 18, 2020

\begin{abstract}
Metformin has been widely used as a therapeutic drug for hyperglycemia and diabetes. Sleep is a vital and restorative process that is necessary for the proper functioning of organs. Sleep deprivation can induce multi-organ injury, including damage to the pancreas and liver that may result in hyperglycemia and diabetes. We studied the role of metformin in reversing sleep deprivation-induced hyperglycemia and pancreatic and liver dysfunction in mice. Mice were kept in cages and fed water and food ad libitum. Mice were subjected to a cycle of 1-day sleep deprivation (7:00 am-7:00 pm) and 1-day sleep for 30 days (15 cycles). Metformin (100 or $300 \mathrm{mg} / \mathrm{kg} /$ day p.o.) was administered from 16th to 30th day. Animals were killed on day 31. The pancreatic function was analyzed by determining the levels of serum glucose, amylase (AMYL), and insulin. Inflammation of the pancreas and liver was investigated by studying the expression of iNOS and NFKB, and levels of cytokines. Proteins involved in the GLUT2-PPARY-PAMPK glycolytic pathway in the liver were analyzed to evaluate the anti-hyperglycemic role of metformin. Sleep deprivation increased blood glucose, AMYL, and GPT levels. Furthermore, it resulted in liver and pancreatic inflammation.
\end{abstract}

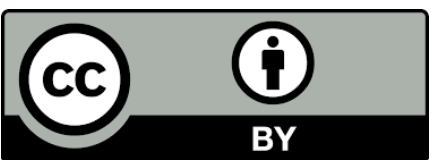


However, compared with control, sleep deprivation decreased the levels of proteins involved in the GLUT2-PPARY-PAMPK glycolytic pathway. Sleep deprivation plus metformin decreased blood glucose and GPT levels and pancreatic inflammation. However, the combination increased the levels of proteins involved in the GLUT2-PPAR -pAMPK glycolytic pathway. In addition, metformin alone increased the levels of AMYL, as well as resulted in islet atrophy, edge irregularities, and disordered pancreatic acinar cells. Metformin attenuates hyperglycemia and reduces pancreatic and liver inflammation in sleep-deprived mice; however, it may cause pancreatic dysfunction.

\section{Keywords}

Sleep deprivation; hyperglycemia; metformin; pancreatic dysfunction; liver dysfunction

\section{Introduction}

Adequate sleep is essential for ideal daytime functioning and overall performance and wellbeing. According to the National Sleep Foundation, the average duration of sleep of Americans has significantly declined in the past century, with a loss of about $1.5 \mathrm{~h}$ per night, from the average 9.0 $\mathrm{h}$ per night in 1910 to the average $7.5 \mathrm{~h}$ per night in 2014. Extended work hours, increased work shifts, and any time availability have resulted in sleep deprivation. The term "bad health" can be defined as the development and progression of preventable chronic diseases, such as obesity, diabetes mellitus, hypertension, and cardiovascular diseases, which may result from sleep deprivation, smoking, excessive alcohol consumption, poor diet, lack of workout, and chronic stress. According to a self-analysis conducted in East Asia, short sleep duration has been rated to cause 30 to $40 \%$ bad health -in Japan and South Korea, and 20 to $30 \%$ in Thailand and Taiwan. Thus, a direct relationship exists between sleep deprivation and bad health such that it has become a potential public health concern [1].

Sleep deprivation induces chronic endoplasmic reticulum stress that leads to necrosis or loss of $\beta$-cells in the pancreas; this eventually exacerbates metabolic loss by decreasing the insulin secretion and results in diabetes [2]. Sleep deprivation includes short term, acute, or decreased sleep and has been reported to be associated with glucose intolerance, insulin resistance, and decreased acute insulin response to glucose, making individuals susceptible to type 2 diabetes [3]. Acute alterations in glucose metabolism are completely reversible by restoring proper sleep. However, chronic sleep loss leads to increasingly harmful effects on the body; and if the continued sleep deprivation is not reversed, it may lead to long-term adverse health consequences [4], ultimately causing circadian disruption and decreased resting metabolic rate and glucose metabolism [5].

Metformin is an anti-glycemic agent commonly used to treat type 2 diabetes mellitus. It is an effective drug if prescribed in the right clinical condition. It decreases the blood sugar levels and controls diabetes; in addition, it lowers the blood triglyceride levels, without causing weight gain or impairing glucose level [6]. However, it may cause few adverse effects, such as reduced hepatic gluconeogenesis and fatty acid oxidation, increased peripheral glucose sensitivity, and decreased intestinal absorption and appetite [6-8]. Approximately $20 \%$ of patients on metformin have 
reported acute side effects such as nausea, abdominal discomfort, metal flavor, and anorexia [6]. Metformin-induced acute pancreatitis is sporadic in beneficial doses and has been ascribed to an inherent toxic mechanism. However, the cumulative dose of metformin has been related to a higher risk of acute pancreatitis [9]. Hypoglycemia may be credited to metformin intake [10], whereas high blood glucose is related to acute pancreatitis, responsible for low insulin production [7]. Furthermore, high doses of metformin might induce pancreatitis, which is attributed to pancreatic toxicity, leading to acinar cell injury and intercellular leakage of digestive enzymes from ductules [11]. The anatomical therapeutic chemical classification system with defined daily doses (DDD) scheme of the World Health Organization (WHO) reports the DDD of metformin as $2000 \mathrm{mg}$ [12]. In the present study, we used $300 \mathrm{mg}$ of metformin per day as the maximum dose; the human equivalent dose (HED) was calculated as $1460 \mathrm{mg}$ according to the method described by Reagan-Shaw et al. (2008), which do not exceed the DDD of human dose [13]. A retrospective and observational study revealed that metformin therapy is associated with better quality and quantity of sleep duration and efficiency [14]; however, it did not provide insights into pancreatic and liver functions that are related to the hypoglycemic effect of metformin. The present study evaluated the role of metformin in sleep deprivation-induced hyperglycemia and pancreatic and liver dysfunction in sleep-deprived mice.

\section{Materials and Methods}

The animal and experimental protocols were conducted according to the nationally approved guidelines. Mice were kept in a biologically measured animal facility on a 12:12 light/dark cycle with food and water available ad libitum. All experiments were permitted by the Ethics Committee at the National Cheng Kung University. Mice were anesthetized using Zoletil-50 (Virbac; Carros, France) (50 mg/kg, i.p.) and sacrificed by $\mathrm{CO}_{2}$ inhalation at the end of the experiments.

\subsection{Reagents}

Most chemicals used in the current study were purchased from Sigma-Aldrich (St. Louis, MO, USA).

\subsection{Animals}

Six- to 7-week old male C57BL/6J mice, weighing 20 to $26 \mathrm{~g}$, were purchased from our institution's Laboratory Animal Center and fed pellet diet and water ad libitum. The mice were kept in a 12-h light/dark cycle and central air conditioning $\left(25{ }^{\circ} \mathrm{C}, 70 \%\right.$ humidity) throughout the experiment. Animal care and experimental protocols were performed following the nationally approved guidelines (IACUC no.104068).

\subsection{Experimental Protocol}

A slightly modified multiple platform method was applied, which uses a REM technique to induce sleep deprivation in mice. An acrylic tank $(40 \times 30 \mathrm{~cm})$ with 12 columns (platforms, $5 \times 3 \mathrm{~cm}$ ) was filled up to $1 \mathrm{~cm}$ with water (Figure 1a). The loss of muscle tone associated with sleep causes the mice to touch the water and wake up. This model does not restrict movement or social isolation. Mice, from the same cage, were placed in an experimental tank from 7:00 am to 7:00 
$\mathrm{pm}$, with water and food ad libitum for 30 days [18]. Animals were divided into five groups of eight animals. Mice in the control group (CTL) were administered only saline orally; mice in the sleep deprivation group (SD) were subjected to sleep deprivation in the experimental tank as mentioned above for 30 days; mice in the SM1 and SM3 groups were subjected to sleep deprivation for 30 days and administered metformin (100 or $300 \mathrm{mg} / \mathrm{kg}$ ) orally for 15 days from 16th to 30th day, and mice in the M3 group were administered only metformin orally (300 mg/kg). We planned our experiment to induce hyperglycemia through sleep deprivation for 15 days and treat mice with metformin from day 16 to day 30 and analyze hyperglycemia and pancreatic function.

\subsection{Blood Collection}

Blood was collected via venipuncture into a serum separation tube and allowed to clot for 20 to $30 \mathrm{~min}$ at room temperature. It was then centrifuged at $15,000 \mathrm{rpm}$ at $4{ }^{\circ} \mathrm{C}$ for $15 \mathrm{~min}$.

\subsection{Assessing Pancreatic and Liver Dysfunction}

Pancreatic and liver dysfunction and injury were assessed using a blood biochemical analyzer (DRI-CHEM 3500s; Fujifilm, Kanagawa, Japan) by measuring the levels of serum amylase (AMYL) and glutamic pyruvic transaminase (GPT). Insulin was measured using a pre-coated 96-well plate from Mercodia ultrasensitive mouse insulin ELISA (Mercodia).

\subsection{Histology}

Pieces of pancreatic and liver tissue from the mice were cut and placed in $10 \%$ formalin. The pancreatic and liver tissue samples were processed and fixed in paraffin blocks and $5 \mu \mathrm{m}$ thick sections were cut. Next, the sections were stained with hematoxylin and eosin (H\&E), and mounted using DePex-Polystyrene (DPX) dissolved in xylene mountant. The tissue sections were examined under a microscope (magnification: 100x) to assess the pathology.

\subsection{Quantification of IL-16, IL-6 and IL-10}

Pancreatic and liver tissues were homogenized in deionized water (1:10; w/v) and centrifuged at $1250 \mathrm{~g}$ for $10 \mathrm{~min}$ at $4{ }^{\circ} \mathrm{C}$. The levels of interleukin (IL)-1 $\beta$, IL-6, and IL-10 in the tissue supernatant were determined using an enzyme-linked immunosorbent assay (ELISA) (R\&D Systems; Minneapolis, MN, USA). The levels were assessed by measuring the absorbance at 450 $\mathrm{nm}$ and extrapolating from a standard curve with a sensitivity limit of $32.5 \mathrm{pg} / \mathrm{mL}$. Protein concentration (pg/mg) in the tissue was determined using a protein assay dye (Bio-Rad Laboratories, Hercules, CA, USA).

\subsection{Immunohistochemistry}

Pancreatic tissue sections were deparaffinized, rehydrated, blocked, and incubated with a recombinant anti-insulin antibody (EPR17359) (ab181547, Abcam, USA. (dilution: 1:50000) overnight at $4{ }^{\circ} \mathrm{C}$ and developed using a rabbit-specific HRP/DAB (ABC) detection IHC kit (Abcam). The stained sections were counterstained with hematoxylin. After mounting using DPX, the slides were examined under a microscope (magnification, $100 \times$ ). 


\subsection{Immunofluorescence}

Liver tissue sections were deparaffinized and rehydrated through a series of graded ethanol and water. Antigen retrieval was performed by heating the sections in a microwave apparatus using a glass container containing $10 \mathrm{mM}$ sodium citrate buffer $(\mathrm{pH} \mathrm{6.0)}$ for $10 \mathrm{~min}$. Next, the sections were cooled at room temperature, incubated in blocking solution ( $3 \%$ bovine serum albumin (BSA) with $0.2 \%$ Tween 20 in phosphate-buffered saline (PBS) for $1 \mathrm{~h}$, followed by overnight incubation at $4{ }^{\circ} \mathrm{C}$ with primary antibodies against pACC [EPR4971] (1:250 dilution, Abcam) and NF-kB [MAB3026] (1:500 dilution, EMD Millipore) diluted in 3\% blocking solution. Next day, the sections were washed and incubated with secondary antibody conjugated to Alexa Fluor 488 (Code: 111-545-003) (1:200 dilution, Jackson ImmunoResearch Laboratories, Inc., Philadelphia, PA, USA) and/or Alexa Fluor 647 (1:200 dilution, ab150115, Abcam) for $1 \mathrm{~h}$ at room temperature in the dark. The sections were washed in PBS and rinsed in distilled water and mounted using fluoroshield mounting medium with 4',6-diamidino-2-phenylindole (DAPI) (Abcam). Images were captured with a microscope (Olympus BX51) under fluorescence setup with appropriate filters.

\subsection{Western Blotting}

Pancreatic and liver cytoplasmic proteins were extracted from the tissues by homogenizing in $200 \mu \mathrm{L}$ of protein lysis buffer. The homogenate was kept on ice for $30 \mathrm{~min}$. After centrifugation (12000 rpm for $30 \mathrm{~min}$ ), the protein concentration in the supernatant was determined by a protein assay dye (Bio-Rad; Hercules, CA, USA) using bovine serum albumin (BSA) as the standard. One hundred $\mu \mathrm{g}$ protein was separated on $10 \%$ SDS-PAGE, and transferred to nitrocellulose sheets (NEN Life Science Products Inc, Boston, MA, USA) with a western blot apparatus (Bio-Rad; Hercules, CA, USA) run at $1.2 \mathrm{~A}$ for $2 \mathrm{~h}$. After blocking in $5 \%$ non-fat skim milk, blots were incubated with rabbit anti-iNOS (1:8000) (Catalog no. 610432, BD Transduction Laboratories, USA), rabbit anti-NF-kB (1:500) (anti-NFKB, p65 subunit, active subunit, clone 12H11, MAB3026, Millipore, USA), rabbit anti-pAMPK (1:1000) (phospho-AMPKa (Thr172) (D4D6D) rabbit mAb Catalog number: 50081, Cell Signaling, USA), rabbit anti-PPARy (1:1000) (PPARy (81B8) rabbit, mAb Catalog number: 2443, Cell Signaling, USA), rabbit anti-GULT2 (1:1000) (GLUT2 rabbit polyclonal antibody, Catalog number: 20436-1-AP, Proteintech, USA), rabbit anti-GCK (1:1000) (GCK rabbit polyclonal antibody, Catalog number: 19666-1-AP, Proteintech, USA), rabbit anti-PFK1 (1:3000) (PFK rabbit polyclonal antibody, Catalog number: 55028-1-AP, Proteintech, USA), rabbit anti-FBP1 (1:20000) (FBP1 rabbit polyclonal antibody, Catalog number: 12842-1-AP, Proteintech, USA), rabbit anti-GAPDH (1:15000) (Monoclonal anti-GAPDH, G8795, Sigma-Aldrich, USA), mouse anti- $\beta$-tubulin (1:1000) (Recombinant anti-beta Tubulin antibody (EPR1330) (ab108342), Abcam's RadMAb technology, USA) antibodies. Following washing with TBST, blots were incubated with anti-mice or anti-rabbit IgG conjugated with alkaline phosphatase (dilution 1:3000) (Jackson ImmunoResearch Laboratories, Inc., Philadelphia, PA, USA). Immunoblots were developed using the BCIP/NBT solution (Kirkegard \& Perry Laboratories Inc., Baltimore, MD, USA). 


\subsection{Statistical Analysis}

All statistical analyses were performed using SPSS 11.0.1 (SPSS Inc., Chicago, IL). Data are expressed as means \pm standard deviation. Differences in the measured variables between each group were assessed using Fisher's least significant difference (LSD) test. Significance was set at $P$ $<0.05$.

\section{Results}

\subsection{Effect of Metformin on Sleep Deprivation-Induced Physiological Changes}

To evaluate the role of metformin during sleep deprivation, we calculated the body weight and food intake. Experimental animals showed no mortality during the course of the study. Bodyweight did not change significantly between the groups during the study. However, food intake was increased in the SD and SM3 groups relative to the CTL group. Food intake in the SM1 group was significantly higher than in all other groups. No change in the food intake was observed in the M3 group compared with the CTL group (data not shown).

\subsection{Effect of Metformin on Sleep Deprivation-Induced Hyperglycemia and Pancreatic Dysfunction}

\subsubsection{Blood Glucose and Insulin}

In order to investigate the protective effect of metformin on the pancreas, serum AMYL, GLU, and insulin levels were quantified. Serum AMYL was significantly $(P<0.01-0.02)$ increased in groups compared with the CTL group. Serum GLU was significantly increased $(P<0.00058)$ in the SD group compared with the CTL group. A significant dose-dependent decrease in the GLU was observed in the SM1 $(P<0.0001)$ and SM3 $(P<0.005)$ groups compared with the SD group. No change in the level of GLU was noticed between the M3 and the CTL groups. However, there were no alterations in the levels of insulin between the experimental groups (Figure $1 \mathrm{~b}$ and $\mathrm{c}$ ).
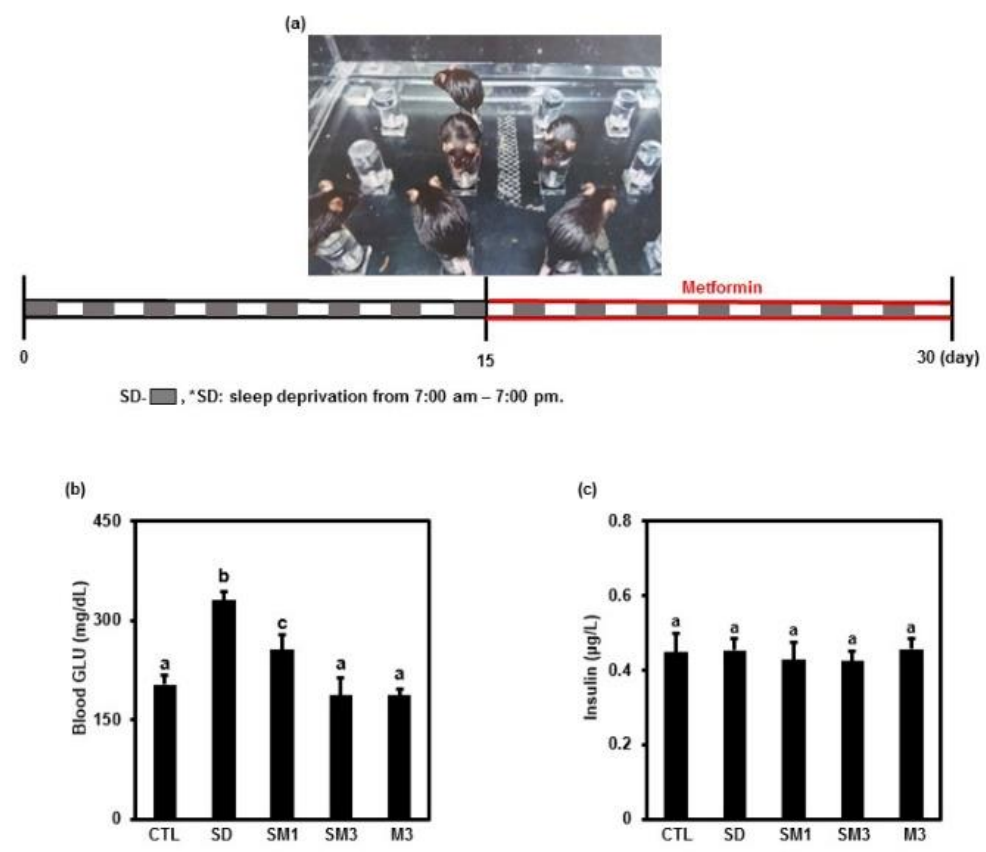
Figure 1 Effect of metformin on sleep deprivation-induced. (a) Experimental protocol, (b) blood GLU, (c) insulin. The control group (CTL) was given saline through gavage. Sleep deprivation (SD) group, SM1 and SM3 groups ( $n=6$, each group) were subjected to sleep deprivation and gavaged with metformin (100 or $300 \mathrm{mg} / \mathrm{kg} / \mathrm{day}$ ) from 15 th to 30th day. M3 group was gavaged only metformin ( $300 \mathrm{mg} / \mathrm{kg} /$ day) from 15th to 30th day. Data expressed as means \pm standard deviation. ${ }^{a}$, ${ }^{6}$ The differences between treatments with different letters are significant $(P<0.05)$.

\subsubsection{Serum Amylase, Histology, and Insulin Immunohistochemistry}

In order to examine the role of metformin on the pancreas, histopathology was performed. Serum amylase was significantly increased in SD $(P<0.014)$, SM1 $(P<0.023)$, SM3 $(P<0.025)$, and M3 $(P<0.024)$ groups compared with the CTL group. Histology revealed mild changes and loss of islets in the pancreas in the SD group compared with the CTL group. However, pancreatic sections in SM1, SM3, and M3 groups showed dose-dependent changes such as islet atrophy, edge irregularities, and disordered acinar cells (Figure $2 a$ and b). Insulin immunohistochemistry revealed normal islets in the CTL group. In the SD group, mild loss of islets with decease in size was observed compared with the CTL group. However, in the SM1 group, pancreas immunohistochemistry showed loss and decreased islet size (Figure 3a and b). In the SM3 and M3 groups, disintegrated islets with loss and size reduction was observed relative to the CTL group.

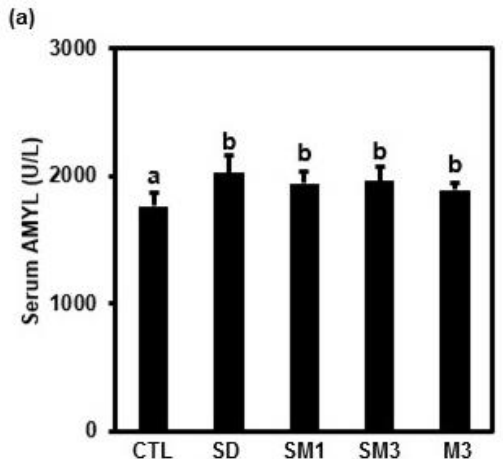

SM1

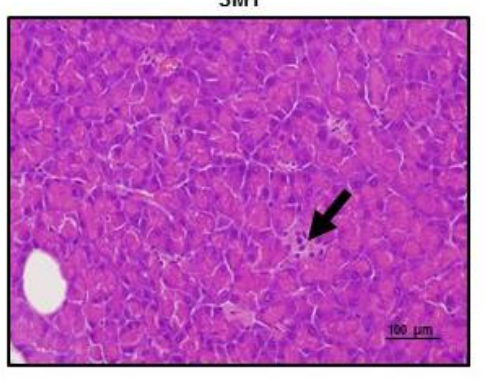

(b)

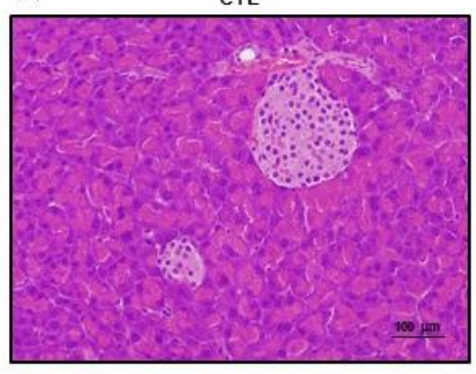

SM3

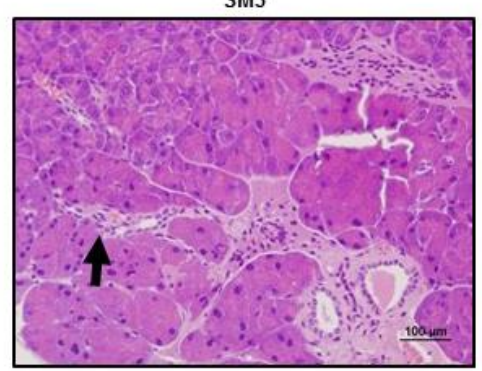

SD

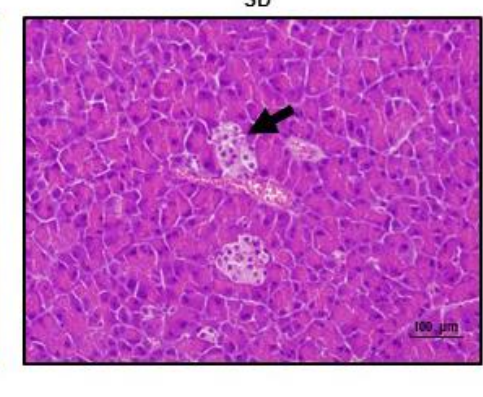

M3

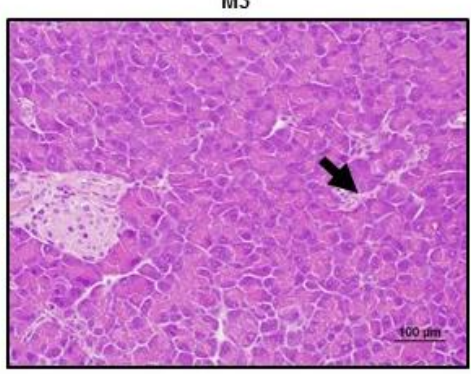

Figure 2 Effect of metformin on sleep deprivation-induced. (a) serum AMYL, (b) pancreatic histopathology. (See groups and treatment details in the legend of Figure 1). Data expressed as means \pm standard deviation. ${ }^{a}$, $b$, 'The differences between treatments with different letters are significant $(P<0.05)$. 
(a)

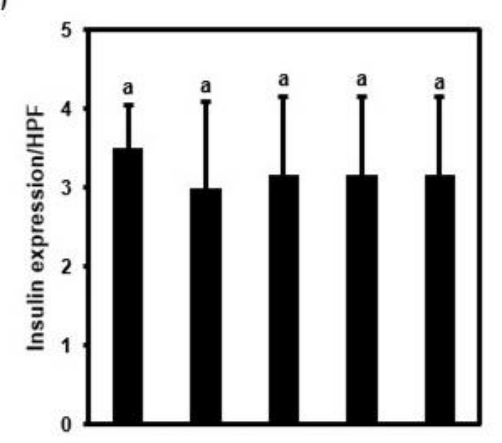

SM1

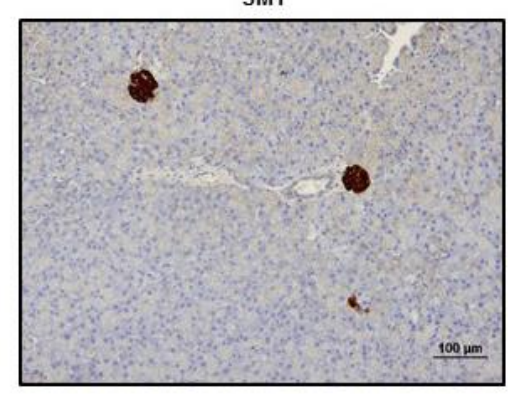

(b)

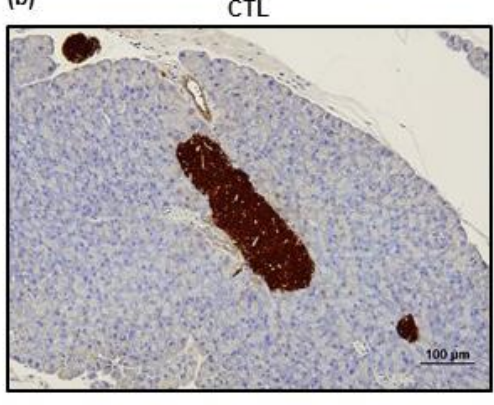

SM3

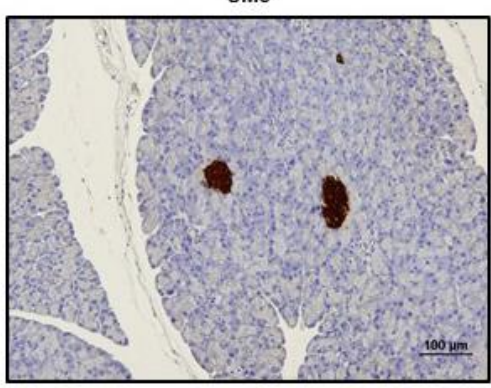

SD

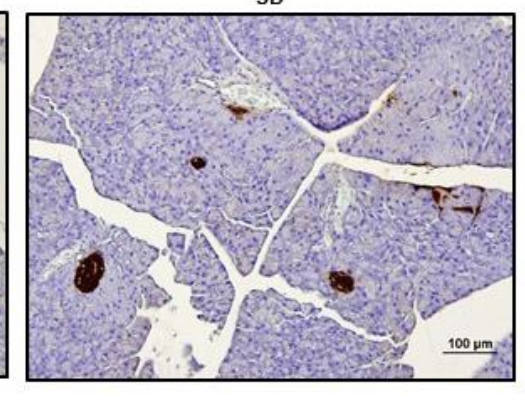

M3

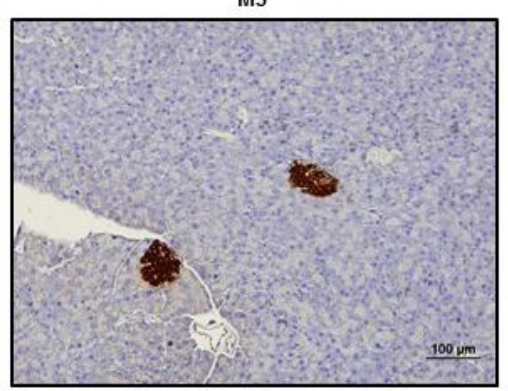

Figure 3 Effect of metformin on sleep deprivation-induced. (a) immune-histochemical score, (b) insulin immune-histochemistry. (See groups and treatment details in the legend of Figure 1). Data are expressed as means \pm standard deviation. ${ }^{a, b}{ }^{c}$ The differences between treatments with different letters are significant $(P<0.05)$.

\subsubsection{Cytokines}

In order to evaluate the effect of metformin on SD-induced inflammatory cytokine, levels of IL$6, I L-1 \beta$, and IL-10 in the pancreatic tissue were assessed. A significant increase in the level of IL- 6 $(P<0.037), I L-1 \beta(P<0.03)$, and IL-10 ( $P<0.037)$ significantly decreased in the SD group compared to the CTL group. The levels of IL-6 (SM1, P < 0.039; SM3, P < 0.0046) and IL-1 $(\mathrm{SM} 3, P<0.05)$ significantly decreased and that of IL-10 in the SM3 group $(P<0.05)$ significantly increased compared with the SD group (Figure 4a-c).

\subsubsection{Expression of iNOS, NFKB, and GLUT2}

In order to examine the effect of metformin on the role of inflammation and glucose transporter, levels of iNOS, NFKB, and GLUT2 were assessed. The levels of iNOS $(P<0.014)$ and NFKB $(P<0.0014)$ significantly increased, and that of GLUT2 $(P<0.0076)$ significantly decreased in the SD group compared with the CTL group. Further, the expression of iNOS (SM1, $P<0.009)$ and NFKB (SM1, $P<0.0027$; SM3, $P<0.004$ ) significantly decreased and that of GLUT2 $r$ remained unchanged in the SM3 group compared with the SD group. The expression of GLUT2 in the SM3 and $\mathrm{M} 3$ groups was significantly higher than that in all other groups (Figure 4d-f). 
(a)

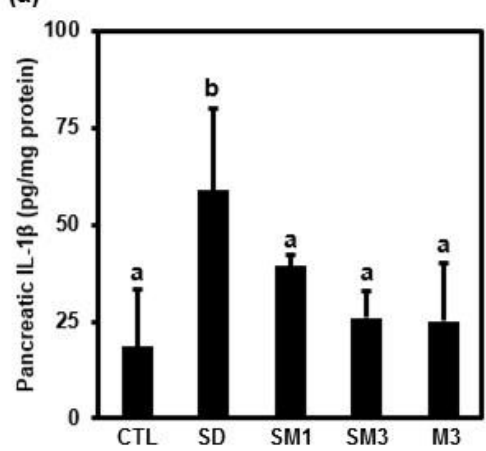

(c)

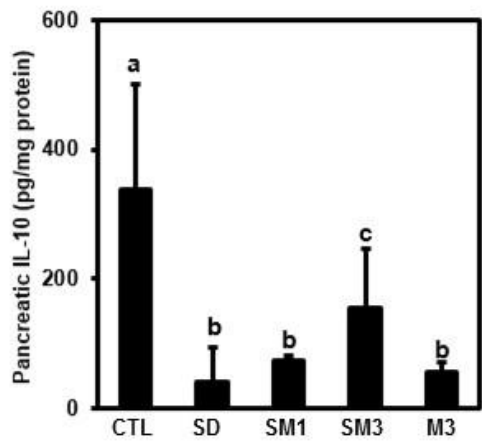

(e)

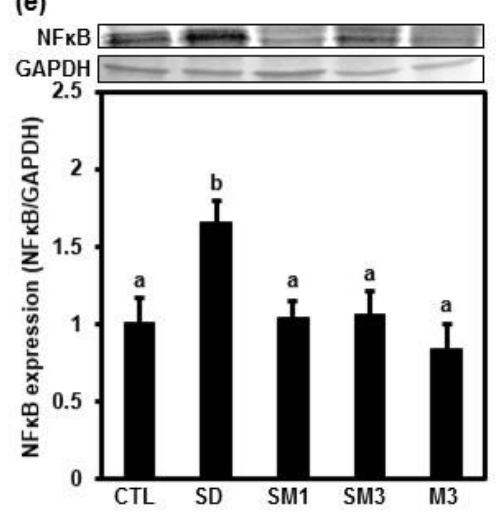

(b)

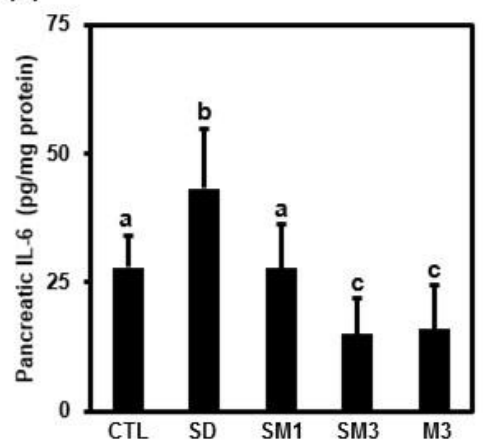

(d)

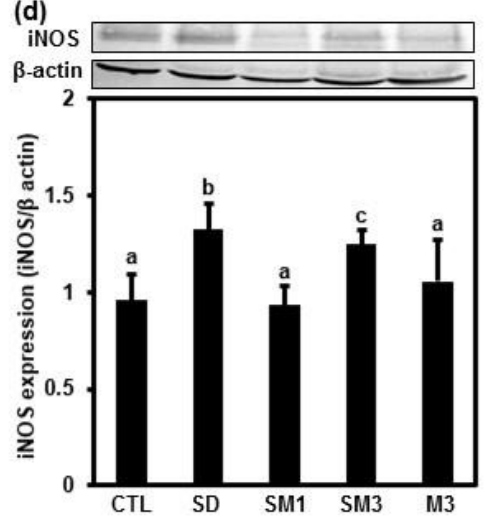

(f)

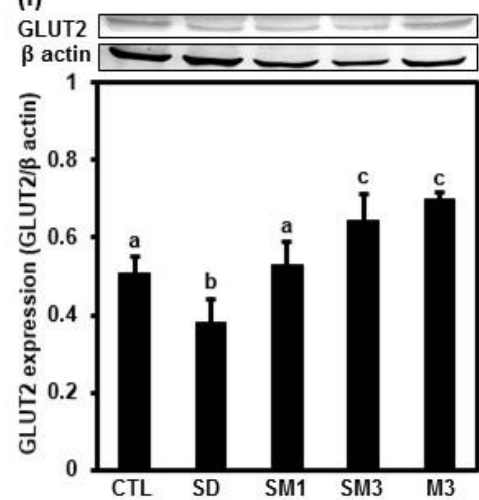

Figure 4 Effect of metformin on sleep deprivation-induced pancreatic. (a) IL-1及, (b) IL-6, (c) IL-10, (d) iNOS expression, (e) NFKB expression and (f) GLUT2 expression. (See groups and treatment details in the legend of Figure 1). Data expressed as means \pm standard deviation. ${ }^{a, b},{ }^{c}$ The differences between treatments with different letters are significant $(P<0.05)$.

\subsection{Effect of Metformin on Sleep Deprivation-Induced Liver Dysfunction, Inflammation and Expression of GLUT2-PPARY-pAMPK Pathway}

\subsubsection{Liver Dysfunction and Histology}

In order to evaluate the effect of metformin on sleep deprivation-induced liver dysfunction, serum GPT levels and histopathology were assessed. A significant increase was observed in the level of GPT $(p<0.027)$ in the SD group compared with the CTL group. A significant dosedependent decrease in the GPT was observed in the SM1 $(P<0.017)$ and SM3 groups compared 
with the SD group. No change in the levels of GPT was noticed between the M3 and the CTL groups (Figure 5a). Histology revealed a mild morphological change in hepatocytes with necrosis and swelling of hepatocytes around the central and portal veins in the SD group. Morphology of hepatocytes in SM1 and SM3 groups showed no significant alteration as compared with the CTL group (Figure 5b).

(a)

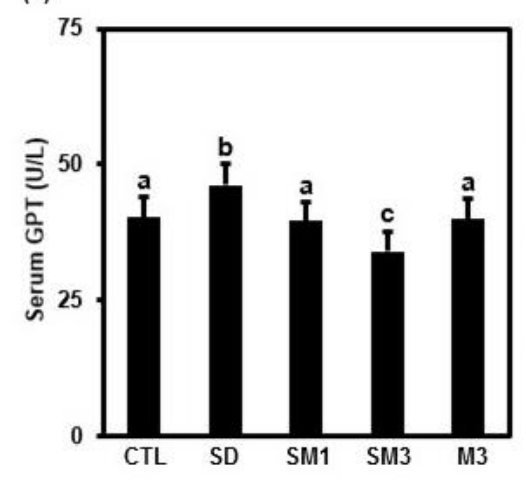

SM1

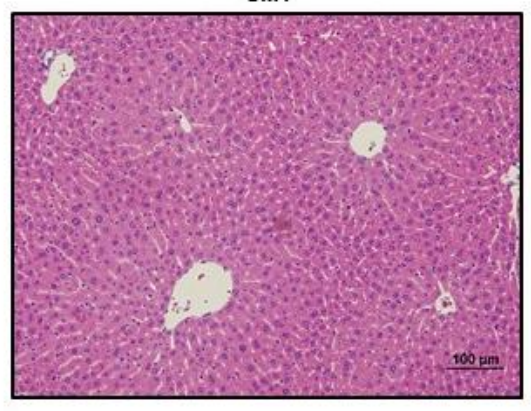

(b)

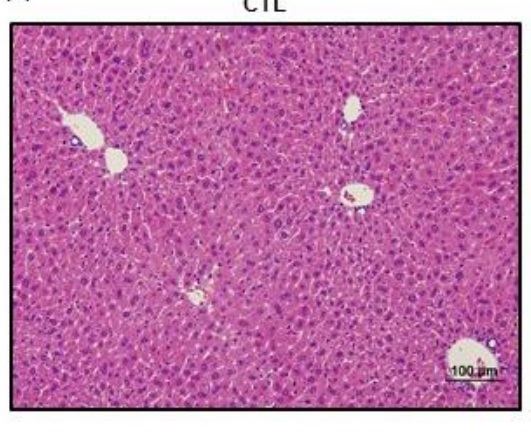

SM3

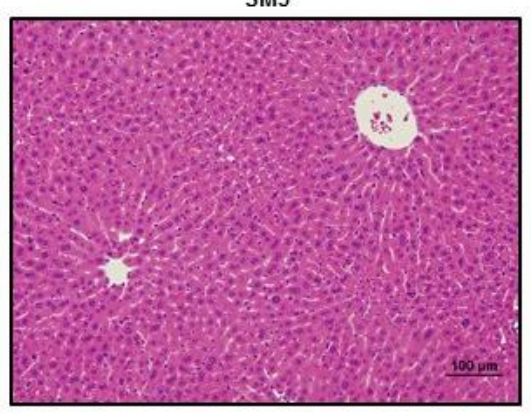

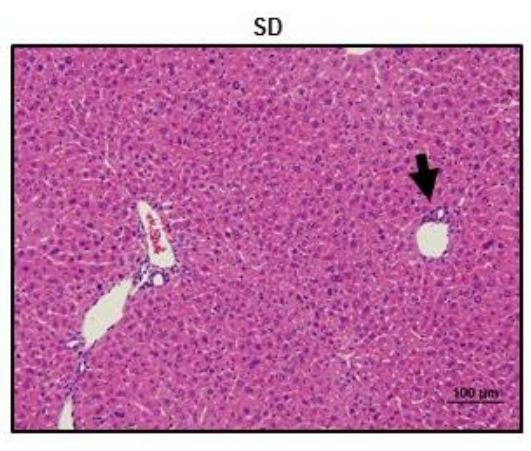

M3

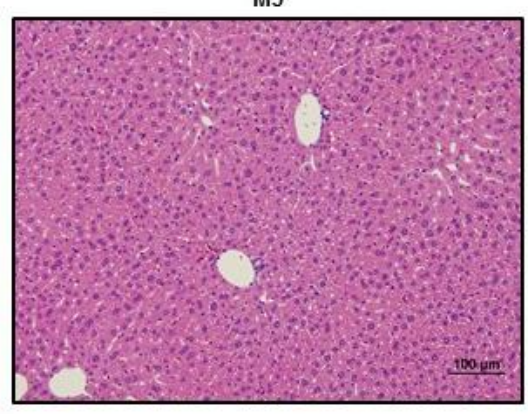

Figure 5 Effect of metformin on sleep deprivation-induced. (a) serum GPT, (b) liver histopathology. (See groups and treatment details in the legend of Figure 1). Data expressed as means \pm standard deviation. ${ }^{a}$, b, c The differences between treatments with different letters are significant $(P<0.05)$.

\subsubsection{Cytokines}

To assess the role of metformin on SD-induced inflammatory cytokines in the liver, IL-6, IL-1 $\beta$, and IL-10 levels were assessed. The level of IL-6 $(P<0.0081)$ and IL-1 $\beta(P<0.00024)$ significantly increased, and that of IL-10 $(P<0.01)$ significantly decreased in the SD group compared with the CTL group. A significant decrease in the levels of IL-6 (SM1, $P<0.022 ; \mathrm{SM} 3, \mathrm{P}=0.019)$ and IL-1 $\beta$ (SM1, $P<0.025$; SM3, $P<0.0021$ ) and IL-10 (SM1, $P<0.015$; SM3, $P<0.019$ ) was significantly increased in the SM1 and SM3 groups as compared with the SD group (Figure 6a-c). 
(a)

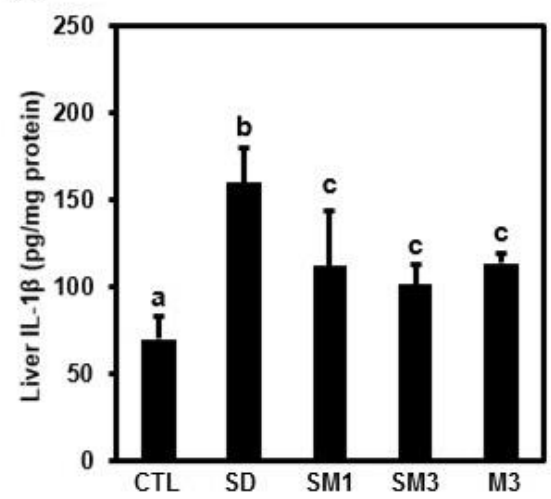

(c)

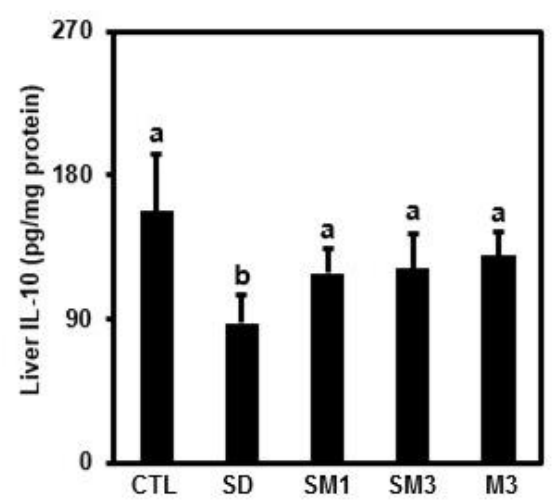

(e)

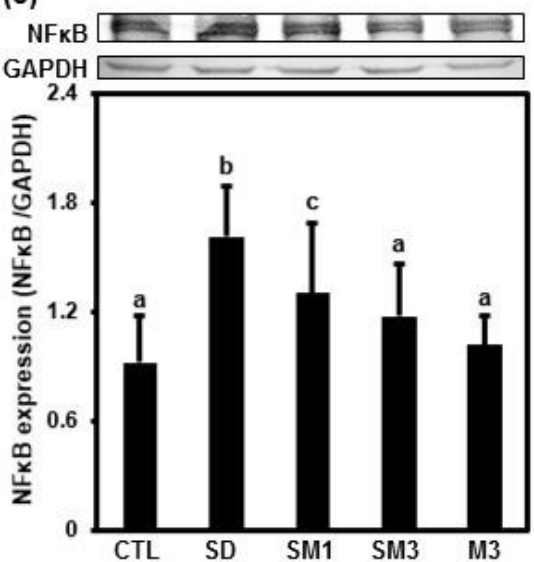

(b)

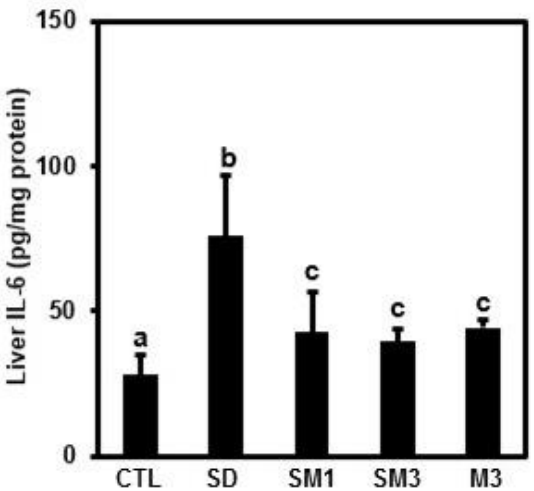

(d)
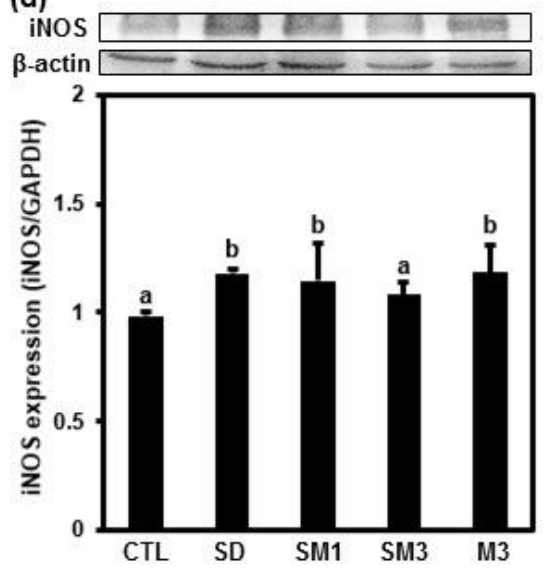

Figure 6 Effect of metformin on sleep deprivation-induced liver. (a) IL-1 $\beta$, (b) IL-6, (c) IL-10, (d) iNOS expression, (e) NFKB expression. (See groups and treatment details in the legend of Figure 1). Data expressed as means \pm standard deviation. ${ }^{a},{ }^{b},{ }^{c}$ The differences between treatments with different letters are significant $(P<0.05)$.

\subsubsection{Expression of iNOS and NFKB}

To examine the effect of metformin on liver inflammation, expression of iNOS and NFkB was assessed. The expression of iNOS $(P<0.00019)$ and NFKB $(P<0.0051)$ significantly increased in the SD group compared with the CTL group. No change in the expression of iNOS was observed in the SM1 group as compared with the SD group. However, the expression of iNOS significantly $(P<$ 
0.0388) decreased in the SM3 group as compared with that in the SD group. The expression of NFKB significantly decreased in the SM3 $(P<0.035)$ group as compared with the SD group. The expression of iNOS in the M3 group significantly increased as compared with the CTL group (Figure $6 \mathrm{~d}$ and e).

\subsubsection{Expression of Liver GLUT2-PPARy-pAMPK Pathway}

To examine the anti-hyperglycemic role of metformin, the expression of GLUT2, PPARY, pAMPK, pACC, GCK, PFK1, and FBP1 in the liver was quantified. The expression of GLUT2 $(P<0.0076)$
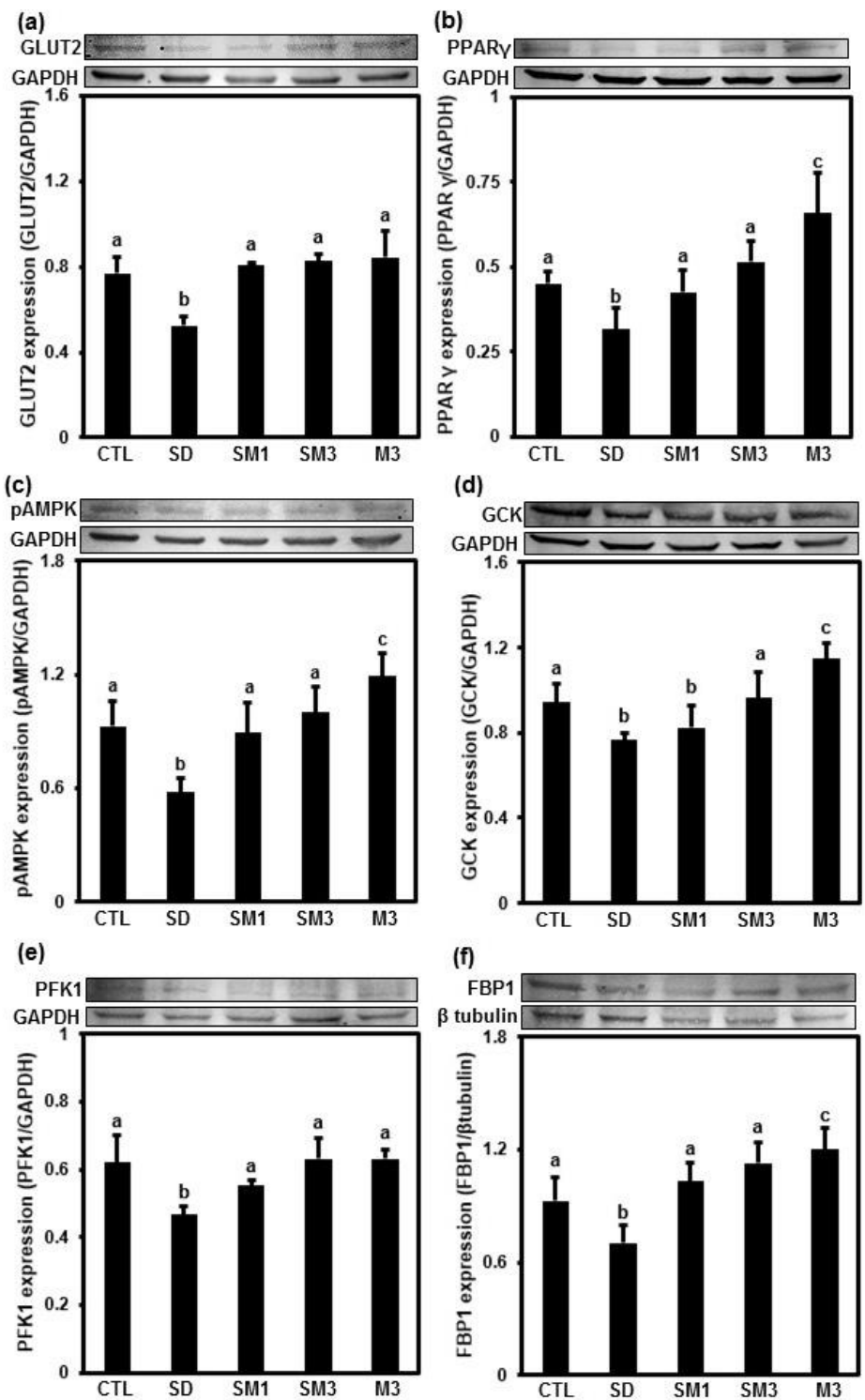

Figure 7 Effect of metformin on sleep deprivation-induced liver. (a) GLUT2 expression, (b) PPARY expression, (c) PAMPK expression, (d) GCK expression, (e) PFK1 expression, (f) FBP1 expression. (See groups and treatment details in the legend of Figure 1). Data expressed as means \pm standard deviation. ${ }^{\mathrm{a}, \mathrm{b}}$, cThe differences between treatments with different letters are significant $(P<0.05)$. 
(Figure 7a), PPARy $(P<0.00019)$ (Figure 7b), pAMPK $(P<0.00095)$ (Figure 7c), GCK $(P<0.0036)$ (Figure 7d), PFK1 $(P<0.013)$ (Figure 7e), FBP1 $(P<0.0088)$ (Figure 7f), and pACC (Figure 8) significantly decreased in SD group as compared with the CTL group. The following GLUT2-PPAR $\gamma$ PAMPK pathway-related proteins were significantly increased in GLUT2 (SM1, $P<0.0022$; SM3, $P<$ 0.00044), PPARY (SM1, $P<0.028$; SM3, $P<0.0022$ ), pAMPK (SM1, $P<0.0018$; SM3, $P<0.0004)$, GCK (SM3, $P<0.019)$, PFK1 (SM1, $P<0.0011$; SM3, $P<0.0037$ ), FBP1 (SM1, $P<0.0007$; SM3, $P<$ $0.0005)$ in the SM1 and SM3 groups as compared with the SD group. In the M3 group, the expression of pAMPK, PPARY, GCK, and FBP1 were significantly elevated than in the CTL group.
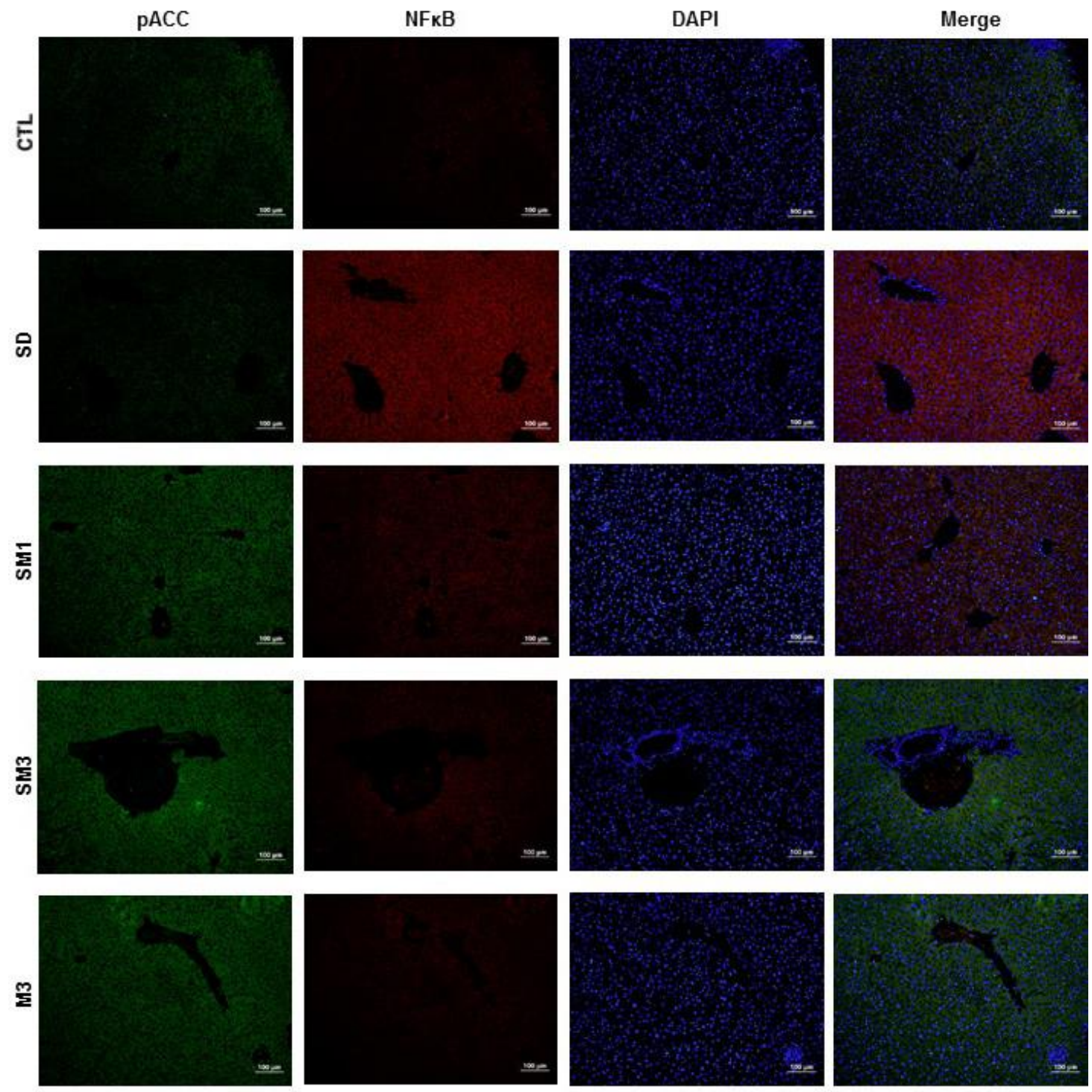

Figure 8 Effect of metformin on sleep deprivation-induced liver pACC-NFkB immunofluorescence. (See groups and treatment details in the legend of Figure 1).

\section{Discussion}

Sleep deprivation and metformin did not alter the blood insulin levels. Metformin decreased the sleep deprivation-induced levels of pancreatic cytokines IL- 6 and IL-1 but increased the level of 
IL-10. Metformin decreased sleep deprivation-induced pancreatic expression of iNOS and NFKB. In addition, it increased the expression of pancreatic GULT2. On the contrary, metformin decreased the levels of GPT, iNOS, NFKB, IL-6, and IL-1 $\beta$, but increased the levels of IL-10 in the liver. With respect to sleep deprivation-induced hyperglycemia, metformin upregulated the expression of GLUT2, PPARY, pAMPK, pACC, GCK, PFK1, and FBP1. We hypothesize that metformin may exert its anti-hyperglycemic action via the activation/expression/phosphorylation of the GLUT2-PPARYPAMPK glycolytic pathway.

Metformin failed to attenuate sleep deprivation-induced pancreatic dysfunction. In addition, pancreatic histopathology revealed the loss of islet cells in most pancreatic sections. Sleep deprivation altered the levels of inflammatory cytokines in both the organs. Metformin treatment had no effect on AMYL-related-pancreatic dysfunction, as reflected from the histopathology that showed loss of islets and hypertrophy. Long-term administration of metformin had no stimulatory effect on inhibition of glucose-stimulated insulin secretion. Moreover, it is known to cause the death of $\beta$ cells by suppressing their proliferation [15]. Furthermore, it has been reported to activate AMPK-induced autophagy in pancreatic $\beta$-cells and enhance the disposal of accumulated autophagic vacuoles in $\beta$-cells [16]. Among other functions of metformin, its anti-inflammatory action has promising clinical implications. Metformin attenuated the sleep deprivation-induced liver dysfunction, evident by reduced GPT levels and liver pathology. Metformin attenuates liver injury by activating the AMPK signaling and reducing oxidative stress [17]. In the present study, metformin attenuated liver dysfunction; however, it failed to attenuate pancreatic dysfunction in sleep-deprived mice.

Metformin attenuated sleep deprivation-induced pancreatic and liver inflammation. Sleep deprivation modulates the number of circulating lymphocytes, natural killer cells, antibody titers, and levels of cytokines, such as IL- 6 and TNF- $\alpha$, ultimately causing immune dysfunction. Sleep deprivation is characterized by higher levels of pro-inflammatory agents, such as TNF- $\alpha$ and IL-6, and a shift toward Th2 activity, as reflected by an altered Th1/Th2 cytokine balance, finally causing inflammation [18]. According to a study, sleep for $4 \mathrm{~h}$ per night for five nights increased the levels of IL-1 $\beta$ and IL- 6 [19]. IL-1 $\beta$ promotes NFKB activation, and in turn, IL-1 $\beta$ production is elevated by NF-kB activation. NF-kB is a transcription factor that acts as an enhancer for TNF- $\alpha$, cyclooxygenase, and iNOS. NF-kB activation plays a key role in sleep deprivation [20], thereby causing pancreatic and liver dysfunction, ultimately causing hyperglycemia. Metformin activates the AMPK signaling pathway to inhibit NF-KB and subsequently reduces the expression of proinflammatory cytokines, such as IL-1 $\beta, \mathrm{IL}-6$, and TNF- $\alpha$, in the pancreas and liver [21-24]. In the present study, metformin attenuated pancreatic and liver inflammation by regulating the expression of cytokines via the AMPK-NF-KB signaling pathway in sleep-deprived mice.

Metformin attenuated sleep deprivation-induced hyperglycemia. Sleep deprivation is known to alter glucose hemostasis or tolerance. Glucose tolerance is the ability of the body to metabolize exogenous glucose and return it to the baseline levels. However, in the present study, elevated fasting glucose was observed during sleep deprivation. Even two days of sleep reduction leads to elevated glucose levels [25]. Sleep deprivation in humans results in decreased glucose tolerance [26]. Most mechanisms of glucose hemostasis or tolerance in sleep deprivation are attributed to the defect in insulin secretion and regulation. However, in the present study, we did not find any alterations in the blood insulin levels. In addition, we found that metformin did not disturb the glucose hemostasis; therefore, the decrease in blood glucose was independent of insulin action. 
Metformin treatment elevated the expression of GLUT2, PPARy, pAMPK, pACC, GCK, PFK1, and FBP1 proteins involved in glycolysis in the liver, thus attenuating hyperglycemia. In sleep deprivation-induced hyperglycemia, the expression of liver GLUT2, PPAR $\gamma$, PAMPK, GCK, PFK1, and FBP1 was diminished, resulting in reduced oxidation of glucose through glycolysis. Blood glucose is taken up by the hepatocyte via the GLUT2, a membrane-bound transporter with high capacity and low affinity for glucose. Next, glucose is phosphorylated to glucose-6-phosphate by GCK, the ratelimiting enzyme for hepatic glucose utilization. The expression and activity of GLUT2 are independent of insulin signaling. In the pancreas, GLUT2 is referred to as a glucose sensor [27]. Metformin exerts its hypoglycemic effect by upregulation of GLUT2 in the liver [28]. Metformin increases the expression of GLUT2 expression in parallel with the expression of PPAR $\alpha$, resulting in significant weight loss, reversed insulin resistance, islet hypertrophy, and alleviated hepatic steatosis [29]. The mechanism of metformin action involves AMPK activation and parallel upregulation of PPARy; these are found to be potential effective therapeutic interventions [30]. Metformin activates PPARY in a manner that is both dependent and independent of phosphorylation of AMPK and ACC in the liver, thus inactivating the ACC activity and increasing fatty acid oxidation and glycolysis [24]. Thus, metformin is recognized as an indirect activator of AMPK, with AMPK is the prime mediator of metformin action. Metformin activates AMPK by promoting AMP accumulation. Metformin elevates AMP levels and decreases CAMP levels and glucose. In addition, it binds to PFK1 and FBP1 in the liver with a high affinity to modulate gluconeogenic and glycolytic enzymes independently via AMPK signaling [31-33]. Metformin treatment elevates the expression of PAMPK and phosphorylates PACC with an associated increase in lactate production, a decrease in glucose production, and an increase in fatty acid oxidation, indicating glucose oxidation via glycolysis [34-36]. Sleep deprivation-induced hyperglycemia is attenuated by metformin via the activation of GLUT2, PPAR, , PAMPK, pACC, GCK, PFK1, and FBP1, ultimately enhancing the glucose oxidation by glycolysis and increasing fatty acid oxidation. Animals treated with metformin alone showed a significant increase in the expression of pancreatic and liver proteins such as GLUT2, PPARY, PAMPK, PACC, GCK, PFK1, and FBP1. This could be attributed to the adverse effects of metformin. However, sleep deprivation, along with metformin decreased these parameters, indicating that mild stress due to sleep deprivation, could be compensated by the antioxidant effect of metformin.

The implication of the present study is that individuals who are sleep deprived due to night shifts, flexible shifts, extended shifts, rotating shifts, or frequent international travel by airline should be alerted about sleep deprivation-related hyperglycemia and glucose intolerance. In particular, individuals susceptible to diabetes or related illness should undergo a routine check for pancreatic and liver dysfunction. In addition, work-related sleep deprivation in people with diabetes and on metformin medication might result in insulin resistance, because of unaltered insulin levels and pancreatic dysfunction in the long term.

\section{Conclusions}

Metformin attenuated hyperglycemia and pancreatic and liver inflammation. In addition, it attenuated liver dysfunction but failed to attenuate pancreatic dysfunction in sleep-deprived individuals. The anti-hyperglycemic mechanism of metformin in sleep deprivation might be 
exerted via oxidation of glucose by glycolysis and through the activation of the GLUT2-PPARYpAMPK-glycolytic pathway.

\section{Author Contributions}

Periasamy S conceived of the presented idea. Chien SP, Hsu C, and Hsu DZ designed and performed the experiments, derived the models and analysed the data. Periasamy $S$ and Hsu DZ wrote the manuscript in consultation with Liu M-Y. All authors discussed the results and contributed to the final manuscript.

\section{Funding}

This study was supported by grant 107-2314-B-006-054-MY3 from the Taiwan Ministry of Sciences and Technology.

\section{Competing Interests}

The authors have declared that no competing interests exist.

\section{References}

1. Cappuccio FP, Miller MA. Sleep and cardio-metabolic disease. Curr Cardiol Rep. 2017; 19: 110.

2. Naidoo N, Davis JG, Zhu J, Yabumoto $M$, Singletary K, Brown M, et al. Aging and sleep deprivation induce the unfolded protein response in the pancreas: Implications for metabolism. Aging Cell. 2014; 13: 131-141.

3. Spiegel K, Tasali E, Leproult R, Van Cauter E. Effect of poor and short sleep on glucose metabolism and obesity risk. Nat Rev Endocrinol. 2009; 5: 253-261.

4. Cohen DA, Wang W, Wyatt JK, Kronauer RE, Dijk DJ, Czeisler CA, et al. Uncovering residual effects of chronic sleep loss on human performance. Sci Transl Med. 2010; 2: 14ra3.

5. Buxton OM, Cain SW, O'Connor SP, Porter JH, Duffy JF, Wang W, et al. Adverse metabolic consequences in humans of prolonged sleep restriction combined with circadian disruption. Sci Transl Med. 2012; 4: 129ra43.

6. Brunton LL, Lazo JS, Parker KL. Goodman and Gilman's: The pharmacological basis of therapeutics. New York: McGraw-Hill; 2001.

7. Suchard JR, Grotsky TA. Fatal metformin overdose presenting with progressive hyperglycemia. West J Emerg Med. 2008; 9: 160-164.

8. Bonsignore A, Pozzi F, Orcioni GF, Ventura F, Palmiere C. Fatal metformin overdose: Case report and postmortem biochemistry contribution. Int J Legal Med. 2014; 128: 483-492.

9. Periasamy S, Hsu DZ, Fu YH, Liu MY. Sleep deprivation-induced multi-organ injury: Role of oxidative stress and inflammation. EXCLI J. 2015; 14: 672-683.

10. Chebane L, Bagheri H, Hillaire-Buys D, Géniaux H, Yahioui N, Laroche, et al. Drug-induced pancreatitis. A review of french spontaneous reports. Rev Med Interne. 2015; 36: 573-578.

11. French LK, Horowitz BZ, Hendrickson RG. Acute metformin overdose. Ann Emerg Med. 2010; 56: $72-73$.

12. Gioia S, Lancia M, Persichini A, Bacci M, Suadoni F. Pancreatitis and metformin: Case-report and review of literature. J. Hepatol Gastroint Dis. 2016; 2: 4. 
13. Pan SW, Yen YF, Kou YR, Chuang PH, Yi-Fong Su V, Feng JY, et al. The risk of TB in patients with type 2 diabetes initiating metformin vs sulfonylurea treatment. Chest. 2018; 153: 1347-1357.

14. Reagan-Shaw $S$, Nihal M, Ahmad N. Dose translation from animal to human studies revisited. FASEB J. 2008; 2: 659-661.

15. Kajbaf F, Fendri S, Basille-Fantinato A, Diouf $M$, Rose $D$, Jounieaux $V$, et al. The relationship between metformin therapy and sleep quantity and quality in patients with Type 2 diabetes referred for potential sleep disorders. Diabet Med. 2014; 31: 577-580.

16. Jiang $Y$, Huang $W$, Wang $J, X u Z, H e ~ J$, Lin $X$, et al. Metformin plays a dual role in MIN6 pancreatic $\beta$ cell function through AMPK-dependent autophagy. Int J Biol Sci. 2014; 10: 268277.

17. Kim J, You YJ. Regulation of organelle function by metformin. IUBMB Life. 2017; 69: 459-469.

18. Dai J, Liu M, Ai Q, Lin L, Wu K, Deng $X$, et al. Involvement of catalase in the protective benefits of metformin in mice with oxidative liver injury. Chem Biol Interact. 2014; 216: 34-42.

19. van Leeuwen WM, Lehto $M$, Karisola $P$, Lindholm $H$, Luukkonen $R$, Sallinen $M$, et al. Sleep restriction increases the risk of developing cardiovascular diseases by augmenting proinflammatory responses through IL-17 and CRP. PLoS One. 2009, 4: e4589.

20. Krueger JM. The role of cytokines in sleep regulation. Curr Pharm Des. 2008; 14: 3408-3416.

21. Han X, Tao YL, Deng YP, Yu JW, Cai J, Ren GF, et al. Metformin ameliorates insulitis in STZinduced diabetic mice. PeerJ. 2017; 5: e3155.

22. Saeedi Saravi SS, Hasanvand A, Shahkarami K, Dehpour AR. The protective potential of metformin against acetaminophen-induced hepatotoxicity in BALB/C mice. Pharm Biol. 2016; 54: 2830-2837.

23. Woo SL, Xu H, Li H, Zhao Y, Hu X, Zhao J, et al. Metformin ameliorates hepatic steatosis and inflammation without altering adipose phenotype in diet-induced obesity. PLoS One. 2014; 9: e91111.

24. Lee WH, Kim SG. AMPK-dependent metabolic regulation by PPAR agonists. PPAR Res. 2010; 2010: 549101.

25. Spiegel K, Tasali E, Penev P, Van Cauter E. Sleep curtailment in healthy young men is associated with decreased leptin levels, elevated ghrelin levels, and increased hunger and appetite. Ann Intern Med. 2004; 141: 846-850.

26. Ip M, Mokhlesi B. Sleep and glucose intolerance/diabetes mellitus. Sleep Med Clin. 2007; 2: 19-29.

27. Bechmann LP, Hannivoort RA, Gerken G, Hotamisligil GS, Trauner M, Canbay A. The interaction of hepatic lipid and glucose metabolism in liver diseases. J Hepatol. 2015; 56: $952-$ 964.

28. Gao L, Wang G, Liu H, Yan C. Effect of combined treatment with clozapine and metformin on fasting blood glucose, insulin level, and expression of the glucose transporter-2 (GLUT2) in Sprague-Dawley rats. Shanghai Arch Psychiatry. 2013; 25: 149-156.

29. Souza-Mello V, Gregório BM, Cardoso-de-Lemos FS, de Carvalho L, Aguila MB, Mandarim-deLacerda CA. Comparative effects of telmisartan, sitagliptin and metformin alone or in combination on obesity, insulin resistance, and liver and pancreas remodelling in C57BL/6 mice fed on a very high-fat diet. Clin Sci (Lond). 2010; 119: 239-250. 
30. Ljubicic V, Jasmin BJ. Metformin increases peroxisome proliferator-activated receptor $y$ Coactivator-1 $\alpha$ and utrophin expression in dystrophic skeletal muscle. Muscle Nerve. 2015; 52: 139-142.

31. Miller RA, Birnbaum MJ. An energetic tale of AMPK-independent effects of metformin. J Clin Invest. 2010; 120: 2267-2270.

32. Miller RA, Chu Q, Xie J, Foretz M, Viollet B, Birnbaum MJ. Biguanides suppress hepatic glucagon signaling by decreasing production of cyclic AMP. Nature. 2013; 494: 256-260.

33. Pernicova I, Korbonits M. Metformin-mode of action and clinical implications for diabetes and cancer. Nat Rev Endocrinol. 2014; 10: 143-156.

34. Richardson MC, Ingarnells $S$, Simonis CD, Cameron IT, Sreekumar R, Vijendren A, et al. Stimulation of lactate production in human granulosa cells by metformin and potential involvement of adenosine 5' monophosphate-activated protein kinase. J Clin Endocrinol Metab. 2009; 94: 670-677.

35. Elia EM, Pustovrh C, Amalfi S, Devoto L, Motta AB. Link between metformin and the peroxisome proliferator-activated receptor $\gamma$ pathway in the uterine tissue of hyperandrogenized prepubertal mice. Fertil Steril. 2011; 95: 2534-2537.e1.

36. Fullerton MD, Ford RJ, McGregor CP, LeBlond ND, Snider SA, Stypa SA. Salicylate improves macrophage cholesterol homeostasis via activation of AMPK. J Lipid Res. 2015; 56: 1025-1033.

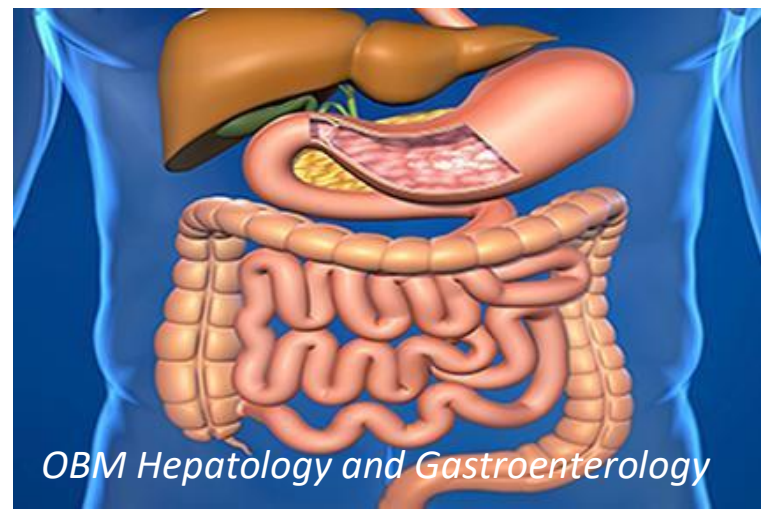

Enjoy OBM Hepatology and Gastroenterology by:

1. Submitting a manuscript

2. Joining in volunteer reviewer bank

3. Joining Editorial Board

4. Guest editing a special issue

For more details, please visit: http://www.lidsen.com/journals/hg 